\title{
Effect of Nursing Intervention on Mental Health and Clinical Effect of Patients with Gastrointestinal Cancer
}

\author{
Lina Wu, Lilian Gao*, Jinfen Han \\ Gastric \& Intestine Department, The First Affiliated Hospital of Jinan University, Guangzhou, China \\ Email address: \\ 1479161420@qq.com (Lina Wu), 943309720@qq.com (Lilian Gao), 542368767@qq.com (Jinfen Han) \\ *Corresponding author
}

\section{To cite this article:}

Lina Wu, Lilian Gao, Jinfen Han. Effect of Nursing Intervention on Mental Health and Clinical Effect of Patients with Gastrointestinal Cancer. Journal of Cancer Treatment and Research. Vol. 7, No. 4, 2019, pp. 77-80. doi: 10.11648/j.jctr.20190704.12

Received: December 1, 2019; Accepted: December 16, 2019; Published: December 26, 2019

\begin{abstract}
Objectives: Following gastrointestinal cancer patients have attracted the attention of the mental health and quality of life in postoperation, we explore outcome associated with nursing intervention improve the mental health and quality of life associated with gastrointestinal cancer patient after chemotherapy by questionnaires. Methods: 78 patients diagnosed as gastrointestinal cancer from March 2018 to October 2019 were randomly assigned to control group and intervention group. We collected the information of mental health and quality of life of patients by Symptom Checklist-90 and Generic Quality of Life Inventory-74. Additionally, the data was analyzed with statistics, $t$ value and $p$ value after collection, which the result can present the changing of mental health and quality of life associated with the nursing intervention. Result: the score of various factors of scl-9 in the intervention group was significantly lower than that in the control group. Additionally, the score of the intervention group was significantly higher than that of the control group. Conclusion: The nursing intervention improve the mental health and quality of life on patients who undergo chemotherapy. In addition, the nursing intervention can improve the outcome of treatment as more patients is willing to cooperate with the treatment arranged by the hospital.
\end{abstract}

Keywords: Gastrointestinal Cancer, Quality of Life, Mental Health

\section{Introduction}

Gastric cancer and colorectal cancer are one of cancers in the digestive tract, its morbidity is high in all kinds of cancers. It presented a trend of becoming younger, posing a great threat to people's health [1]. Anxiety, depression and psychological distress are common psychological problems in patients with gastrointestinal cancer [2]. Gastrointestinal cancer has no obvious symptoms in the early stage, which can significantly affect the patient's dietary habit after treatment. It is prone to recurrence. Despite the continuous improvement of clinical treatment effect, it still has a high fatality rate $[3,4]$.

Gastrointestinal cancer is one of the malignant tumors of digestive tract, the incidence rate was high with the 2 nd in China and the 5th in the world [5,6]. According to the 2016 annual report of Chinese tumor registration, the incidence of gastric cancer was high in the third among malignant tumors, with a total mortality rate of 245,300/100,000 [7]. Following the reports, most gastrointestinal cancer patients had obvious anxiety, depression mood and poor quality of life before and after treatment $[8,9]$. Unfortunately, psychological factors are the leading deaths of cancer. It accounted about $40 \%$ of all deaths, and depression reduces survival of cancer patients from $10 \%$ to $20 \%[10,11]$.

\section{Samples and Methods}

\subsection{Patients Enrollment}

We investigated the patients who were diagnosed with gastrointestinal cancer. The patients $(n=78)$, their diagnosis of time from March 2018 to October 2019, were included in the control group $(n=39)$ and intervention Group $(n=39)$ by random allocation. Also, the patients would finish the Symptom Checklist-90 (SCL-90) [12] and Generic Quality of Life Inventory-74 (GQOLI-74) [13] in before treatment and after treatment. The SCL-90 include nine factors, such as sensation, emotion, thinking, consciousness and behavior. The patients make a score between 1 to 5 for every factor, the 
higher score means worse mental condition of patients. Besides, GQOLI-74 evaluates material life state, physical function, psychological function and social function on patients, the score standard is from 1 to 5 points, the score is higher as the better the quality of life.

Their inclusion criteria were: (1) was diagnosed with gastrointestinal cancer; (2) agree to set up a personal information account in the First Affiliated Hospital of Jinan University; (3) have the plan to treat gastrointestinal cancer by chemotherapy in this hospital; (4) agreed to participate in this study. Their withdraw criteria were: (1) have serious heart, liver and kidney diseases, so not suitable for drug treatment; (2) cannot complete the questionnaire survey. In addiction, this study was reviewed and approved by the ethics committee of the college.

\subsection{Survey Method}

The control group: doctors and nurses provide related chemotherapy and usual care in duration of hospital stay.

The intervention group: basic on related chemotherapy and usual care, we provided extra nursing intervention to the participants in duration of hospital stay and post-discharge. The extra nursing intervention associated with this study contains: (1) Primary nursing, we not only provided isolation ward to the participants but also use One-to-one Care Model for the participants which is different for post care model of hospital. In particular, the patients are cared for by only one nurse, not by a rotating group of nurses. The nurses going to know the detail associated with patients, such as personality, family situation and relationship. Furthermore, the nurses make a good relationship between patient and hospital by some communication methods. (2) Mental intervention, most of the patients are in a state of anxiety and depression, and some even refuse to receive treatment. So we improved the psychological state of patients by diverting attention and channeling emotions, and we encourage family members and friends to visit the patient actively, and give the patient spiritual support from the family. (3) Behavioral interventions, we will make the patient's recovery plan, such as personal hygiene, taking exercise, healthy life plan and supervising patients' timely treatment.

\subsection{Statistical Analysis}

We performed the statistical analysis by SPSS24.0, The mean standard deviation (measurement data) for statistical description Besides, we compared and analyzed the data of two groups by t-text and chi-square test.

\section{Result}

This study enrolled 78 patients [51 men (65.4\%), 27 women $(34.6 \%)]$ with a mean age of $43.22 \pm 13.15$ years (Mean \pm SD) (Table 1). In marital status of patients, most patients were married, they had an average $19.2 \%$ unmarried $(n=15)$, average $71.8 \%$ married $(n=56)$ and average $9.0 \%$ divorce $(\mathrm{n}=7)$. Also, the education level associated with patients had $24.4 \%$ junior high or lower $(n=19), 35.9 \%$ high school $(n=28)$ and $39.8 \%$ bachelor degree or above $(n=31)$.

Table 1. Participants Characteristics.

\begin{tabular}{ll}
\hline Characteristics & \\
\hline Female n (\%) & $27(34.6 \%)$ \\
Male n (\%) & $51(65.4 \%)$ \\
Age (years) & $43.22 \pm 13.15$ \\
Marital status & \\
Unmarried & $15(19.2 \%)$ \\
Married & $56(71.8 \%)$ \\
Divorce & $7(9.0 \%)$ \\
Education level & \\
Junior high or lower & $19(24.4 \%)$ \\
High school & $28(35.9 \%)$ \\
Bachelor degree or above & $31(39.8 \%)$ \\
\hline
\end{tabular}

The score results of each factor of SCL-90 is shown in Table 2. Before the intervention, there had not significant difference in the score of various factors of scl-9 between the two groups of gastrointestinal cancer patients $(P>0.05)$. After the intervention, the score of various factors of scl-9 in the intervention group was significantly lower than that in the control group.

Table 2. The score results of each factor of SCL-90 in before the intervention and after the intervention (Mean $\pm S D$ points).

\begin{tabular}{|c|c|c|c|c|c|c|c|c|}
\hline \multirow[b]{2}{*}{ Projects } & \multicolumn{4}{|c|}{ Before the intervention } & \multicolumn{4}{|c|}{ After the intervention } \\
\hline & Intervention Group & Control Group & $\begin{array}{l}T \\
\text { Value }\end{array}$ & $\begin{array}{l}P \\
\text { Value }\end{array}$ & Control Group & Intervention Group & T Value & P Value \\
\hline Somatization & $2.21 \pm 0.29$ & $2.17 \pm 0.29$ & 0.46 & $>0.05$ & $1.66 \pm 0.15$ & $1.78 \pm 0.16$ & 3.01 & $>0.05$ \\
\hline Force & $1.88 \pm 0.27$ & $1.91 \pm 0.28$ & 0.36 & $>0.05$ & $1.64 \pm 0.22$ & $1.71 \pm 0.15$ & 2.18 & $>0.05$ \\
\hline Relationship & $1.93 \pm 0.23$ & $1.91 \pm 0.25$ & 0.46 & $>0.05$ & $1.72 \pm 0.18$ & $1.84 \pm 0.27$ & 2.48 & $>0.05$ \\
\hline Depression & $2.25 \pm 0.31$ & $2.21 \pm 0.22$ & 0.52 & $>0.05$ & $1.67 \pm 0.21$ & $1.87 \pm 0.29$ & 3.46 & $>0.05$ \\
\hline Anxiety & $1.92 \pm 0.50$ & $1.90 \pm 0.51$ & 0.41 & $>0.05$ & $1.63 \pm 0.31$ & $1.77 \pm 0.22$ & 2.42 & $>0.05$ \\
\hline Hostile & $1.95 \pm 0.12$ & $1.97 \pm 0.21$ & 0.36 & $>0.05$ & $1.62 \pm 0.42$ & $1.81 \pm 0.33$ & 3.24 & $>0.05$ \\
\hline Fear & $1.50 \pm 0.42$ & $1.47 \pm 0.12$ & 0.44 & $>0.05$ & $1.24 \pm 0.51$ & $1.31 \pm 0.36$ & 2.25 & $>0.05$ \\
\hline Paranoid & $1.77 \pm 0.36$ & $1.75 \pm 0.18$ & 0.54 & $>0.05$ & $1.54 \pm 0.23$ & $1.64 \pm 0.28$ & 2.55 & $>0.05$ \\
\hline Psychotic & $1.54 \pm 0.34$ & $1.57 \pm 0.47$ & 0.43 & $>0.05$ & $1.33 \pm 0.19$ & $1.45 \pm 0.32$ & 2.33 & $>0.05$ \\
\hline
\end{tabular}

SCL-90=Symptom Checklist -90 .

The Table 3 shown the score results of each factor of GQOLI-74. The score of various factors associated with
GQOLI-74 had not significant difference between the two groups. After the intervention, the score of the intervention 
group was significantly higher than that of the control group, the differences were statistically significant $(\mathrm{P}<0.05)$.

Table 3. The score results of each factor of GQOLI-74 in before the intervention and after the intervention (Mean $\pm S D$ points).

\begin{tabular}{llll}
\hline Projects & Cases (n) & Before the intervention & After the intervention \\
\hline Intervention Group & 39 & $168.12 \pm 12.06$ & $195.44 \pm 17.03$ \\
Control Group & 39 & $169.77 \pm 21.23$ & $182.77 \pm 16.11$ \\
T Value & & 0.42 & 3.86 \\
P Value & & $>0.05$ & $<0.05$ \\
\hline
\end{tabular}

GQOLI-74=Generic Quality of Life Inventory-74.

\section{Discussion}

For similar reports, suitable nursing intervention significant improved anxiety, depression and some negative emotions on gastrointestinal cancer patient [14-16]. Comprehensive nursing intervention is on the basis of conventional nursing care including psychology, behavior and family intervention measures, such as the personal nursing model. In the whole treatment process, it not only emphasize the good doctor-patient relationship and importance to disease cognition degree to improve the treatment compliance. Besides, we pay attention to patients with positive incentives, good mental state, behavior of the value of the physical and mental diseases.

In this study, 78 cases of gastrointestinal cancer patients with different nursing methods presents that comprehensive nursing intervention than routine nursing. The nursing intervention effectively reduce the score of each factor of the symptom self-rating scale SCL-9 for patients with gastrointestinal cancer and improve the gqoli-74 score, especially significantly affecting somatization, depression and hostile factors. The result further indicates that comprehensive nursing intervention is conducive to improving the negative mood of drug-addicted patients with tuberculosis and improving their quality of life.

\section{Conclusion}

In conclusion, comprehensive nursing intervention not only helps to improve the mental health status and quality of life of patients with gastrointestinal cancer, improve the treatment compliance and cure rate of patients, but also helps to establish a good doctor-patient relationship so as to improve the medical image. Additionally, the nursing intervention can improve the outcome of treatment as more patients is willing to cooperate with the treatment arranged by the hospital. In the future, Chinese hospital require better nursing intervention to help patients to better recovery. However, Chinese nursing intervention skills require to be improved continuously, because this improvement is worse than some related improvement in report.

\section{References}

[1] Wanqing C, Rongshou Z, Tingting Z, et al. National cancer incidence and mortality in China 2012 [J]. Chinese Journal of Cancer Research, 2016, 28 (1): 1-11.
[2] Dunn J, Ng SK, Holland J, et al. Trajectories of psychological distress after colorectal cancer [J]. Psycho-Oncology, 2013, 22 (8): 1759-1765.

[3] Feng W, Zhengjin M, Shaomin W. Expression of abnormal tumor protein/abnormal sugar chain glycoprotein in serum before and after treatment of rectal cancer [J]. Colorectal surgery, 2016, 22 (2): 150-153.

[4] Tantoy I Y, Dhruva A, Cataldo J, et al. Differences insymptom occurrence, severity, and distress ratings between patients with gastrointestinal cancers who received chemo-therapy alone or chemotherapy with targeted therapy $[\mathrm{J}]$. J Gastrointest Oncol, 2017, 8: 109e126.

[5] Layke JC. Loperz PP. Gastric cancer: diagnosis and treatment options [J]. Chinese journal of general practice, 2015, 18 (3): 248-249.

[6] Piazuelo MB, Correa P. Gastric cancer: overiew [J]. Colomb Med, 2013, 44 (3): 192-201.

[7] 2013 annual report of Chinese tumor registration [EB/OL] (2014-04-19). http//newsXinhua]WaldJ, Taylor, S, Asmundson, GLiterature review of concepts: Psycholo-gical resliency. Toronto (ON): Defence R\&D Canada, 2006.

[8] Cuiyun W, Wili W, Miao W. Quality of life investigation and influencing factors analysis of 105 patients with colorectal cancer [J]. Chinese journal of nursing, 2012, 19 (2): 12-15.

[9] Limei M, Yuxia L. Investigation and effect of psychological intervention on anxiety and depression in patients with gastric cancer [J]. Western medicine, 2013, 25 (6): 867-868, 870.

[10] Huiyi L, Guixiang L, Xuewei H. Psychological characteristics of long-term cancer survivors [J]. Journal of behavioral medicine and brain sciences, 2012, 21 (11): 994-996.

[11] Montgomery K L, Kim J S, Franklin C. Acceptance and commitment therapy for psychological and phychological and physiological illnesses: a systematic review for scial workers [J]. health Sco Work, 2011, 36 (3): 169-181.

[12] Derogatis L R, Savitz K L. The SCL-90-R and the Brief Symptom Inventory (BSI) in Primary Care. In: Maruish, M. E. (Ed.), Handbook of Psychological as-sessment in Primary Care Settings. Lawrence Erlbaum Associates, Mahwah [J], 2000, 217-334.

[13] Lu L, et al. Quality of life in Chinese patients with schizophrenia: A meta-analysis. Psychiatry Research [J], 2018, 268, 392-399.

[14] Westman B, Ullgren H, Olofsson A, Sharp L. Patient-reported perceptions of care after the introduction of a new advanced cancer nursing role in Sweden [J]. European Journal of Oncology Nursing, 2019, 41: 41-48. 
[15] Tantoy I Y, Cataldo J K, Aouizerat B E, Dhruva A, Miaskowski C. A review of the literature on multiple co-occurring symptoms in patients with colorectal cancer who received chemotherapy alone or chemotherapy with tar-geted therapies. Cancer Nurs, 2016, 39: 437-445.
[16] Han C J, Reding K, Cooper B A, et al. Symptom clustersin patients with gastrointestinal cancers using different di-mensions of the symptom experience [J]. J Pain Smptom Manage, 2019, 58: 224-234. 